\begin{tabular}{|c|l|}
\hline Title & Zero-modes on orbifolds: Magnetized orbifold models by modular transformation \\
\hline Author(s) & Kobay ashi, Tatsuo; Nagamoto, Satoshi \\
\hline Citation & $\begin{array}{l}\text { Physical Review D, 96/9), 96011 } \\
\text { https://doi.org/10.1103/PhysRevD.96.096011 }\end{array}$ \\
\hline Issue Date & 2017-11-14 \\
\hline Doc URL & http://hdl.handle.net/2115/67915 \\
\hline Rights & @2017 A merican Physical Society \\
\hline Type & article \\
\hline File Information & PhysRevD96 096011.pdf \\
\hline
\end{tabular}

Instructions for use 


\title{
Zero-modes on orbifolds: Magnetized orbifold models by modular transformation
}

\author{
Tatsuo Kobayashi and Satoshi Nagamoto \\ Department of Physics, Hokkaido University, Sapporo 060-0810, Japan
}

(Received 4 October 2017; published 14 November 2017)

\begin{abstract}
We study $T^{2} / Z_{N}$ orbifold models with magnetic fluxes. We propose a systematic way to analyze the number of zero-modes and their wave functions by use of modular transformation. Our results are consistent with the previous results, and our approach is more direct and analytical than the previous ones. The index theorem implies that the zero-mode number of the Dirac operator on $T^{2}$ is equal to the index $M$, which corresponds to the magnetic flux in a certain unit. Our results show that the zero-mode number of the Dirac operator on $T^{2} / Z_{N}$ is equal to $\lfloor M / N\rfloor+1$ except one case on the $T^{2} / Z_{3}$ orbifold.
\end{abstract}

DOI: 10.1103/PhysRevD.96.096011

\section{INTRODUCTION}

Superstring theory is a promising candidate for unified theory including gravity and leads to six-dimensional space in addition to our four-dimensional (4D) spacetime. Thus, extra dimensional models are well-motivated. Indeed, many studies have been carried out. It is a key point how to derive 4D chiral theory starting from extra dimensional theories, because the standard model is a chiral theory. For example, the toroidal compactification is one of the simplest compactifications, but leads to nonchiral theory. Then, the simple toroidal compactification is not realistic. However, the torus compactification with magnetic fluxes can lead to 4D chiral theory from extra dimensional theories as well as superstring theories [1-4]. In addition, the magnitude of magnetic flux determines the number of zero-modes, which would correspond to the generation number. Also zeromode profiles are quasilocalized and can lead to suppressed couplings depending on their localized points. Hence, the torus compactification with magnetic fluxes is quite interesting. Indeed, several studies have been done, e.g. on computation of Yukawa couplings [5], higher order couplings [6], non-Abelian flavor symmetries [7,8], massive modes and their phenomenological effects [8-11], etc. ${ }^{1}$

The orbifold models with magnetic fluxes are also interesting. Orbifolding can project out the adjoint matter fields corresponding to open string moduli, which remain massless in the toroidal compactification with magnetic fluxes. The number of zero-modes and their profiles in orbifold models are different from those in toroidal models [14]. Thus, orbifold models with magnetic fluxes have rich structures in model building. Indeed, $Z_{2}$ orbifold models have been studied on several aspects, e.g. model building [15-19], realization of quark and lepton masses and their mixing angles and CP phase [20-23]. In addition, it is possible to introduce some degree of freedom on orbifold

\footnotetext{
${ }^{1}$ See also [12,13].
}

fixed points, e.g. localized modes and localized operators. That makes phenomenological aspects richer [24-27].

Other $Z_{N}$ orbifold models with $N=3,4,6$ have been also studied. Zero-mode wave functions were studied by numerical studies [28] and the corresponding states were studied by operator analysis in quantum mechanism [29]. By use of those results, model building and fermion mass matrices were also studied [30-32]. However, the numerical study is not analytical, and results from both approaches were rather complicated. A simpler approach would be useful for further applications.

Here, we study $Z_{N}$ orbifold models with magnetic fluxes. In particular we study the number of zero-modes and their wave functions directly by using modular transformation. The modular transformation is a geometrical transformation of the lattice which is used to construct $T^{2}$. Zero-mode wave functions can be written in terms of theta functions, which have a characteristic behavior under modular transformation. When we fix a value of complex structure properly, certain modular transformation behaves as $Z_{N}$ twists with $N=3,4,6$. Using such behavior, we can obtain zero-mode wave functions on $Z_{N}$ orbifolds. For generic values of magnetic flux, we compute the number of zero-modes with each $Z_{N}$ eigenvalue on $T^{2} / Z_{N}$. We show that the number of $Z_{N}$ invariant zero-modes is almost universal on different $T^{2} / Z_{N}$ orbifolds, and it is equal to $\lfloor M / N\rfloor+1$ for magnetic flux $M$ in a certain unit except one case in the $T^{2} / Z_{3}$ orbifold, where $\lfloor r\rfloor$ denotes the maximum integer $n$ satisfying $n \leq r$. Alternatively, the number of $Z_{3}$ invariant zero-modes is written by $2\lfloor M /(2 N)\rfloor+1$.

This paper is organized as follows. In Sec. II, we review wave functions on the two-dimension torus $T^{2}$ with magnetic fluxes as well as the $T^{2} / Z_{2}$ orbifold. In Sec. III, we study the $T^{2} / Z_{4}$ orbifold. In Sec. IV we study the $T^{2} / Z_{3}$ orbifold as well as $T^{2} / Z_{6}$ orbifold. In Sec. V, we give a comment on our universal result on the number of $Z_{N}$ invariant zero-modes. Section VI is the Conclusion. In Appendix A, we show computations on the normalization 
factor of zero-mode wave function and inner product of two types of wave functions. Such computations are useful for Secs. III and IV. In Appendix B, we show the computation on products of the $Z_{3}$ matrix. In Appendix $C$, we show explicitly zero-mode wave functions on the $T^{2} / Z_{4}$ orbifold.

\section{TORUS MODEL WITH MAGNETIC FLUX}

Our starting point is the gauge theory with $2 n$ extra dimensions, which are chosen as $\left(T^{2}\right)^{n}$. Our theory includes the spinor field $\lambda$, and its Lagrangian is written by

$$
\mathcal{L}=-\frac{1}{4 g^{2}} \operatorname{Tr} F^{M N} F_{F M}-\frac{i}{2 g^{2}} \bar{\lambda}^{M} D_{M} \lambda
$$

where $F_{M N}=\partial_{M} A_{N}-\partial_{N} A_{M}$. Here, we set the kinetic term of $\lambda$ as one in super Yang-Mills theory, because we are motivated from such a theory. For simplicity, we concentrate on U(1) gauge theory with $n=1$ and spinor field with charge $q$. Similarly, we can extend our analysis to a non-Abelian gauge theory with $n \geq 1$.

We decompose

$$
\lambda\left(x^{\mu}, y^{m}\right)=\sum_{n} \eta_{n}\left(x^{\mu}\right) \otimes \psi_{n}\left(y^{m}\right),
$$

where $x^{\mu}$ denotes coordinates of four-dimensional spacetime, while $y^{m}$ with $m=1,2$ denotes coordinates on $T^{2}$. $\psi_{n}\left(y^{m}\right)$ are eigenfunctions of a Dirac operator on $T^{2}$. In what follows, we concentrate on the zero-modes, $\psi_{0}(y)$, which correspond to massless modes in 4D effective field theory, and we denote them by $\psi(y)$.

\section{A. Magnetized torus models}

Here, we give a review on the $T^{2}$ model with a magnetic flux, in particular zero-mode wave functions [5]. We use the complex coordinate $z=y^{1}+\tau y^{2}$ instead of the real coordinates, $y^{1}$ and $y^{2}$, where $\tau$ is a complex, and the metric is given as $d s^{2}=g_{\alpha \beta} d z^{\alpha} d \bar{z}^{\beta}$,

$$
g_{\alpha \beta}=\left(\begin{array}{ll}
g_{z z} & g_{z \bar{z}} \\
g_{\bar{z} z} & g_{\bar{z} \bar{z}}
\end{array}\right)=(2 \pi R)^{2}\left(\begin{array}{cc}
0 & \frac{1}{2} \\
\frac{1}{2} & 0
\end{array}\right) \text {. }
$$

To realize the $T^{2}$, we identify $z \sim z+1$ and $z \sim z+\tau$.

We consider the U(1) magnetic flux $F$ on $T^{2}$,

$$
F=i \frac{\pi M}{\operatorname{Im} \tau}(d z \wedge d \bar{z}) .
$$

Such a magnetic flux can be obtained from the following vector potential:

$$
A(z)=\frac{\pi M}{\operatorname{Im} \tau} \operatorname{Im}(\bar{z} d z)
$$

It satisfies the boundary conditions,

$A(z+1)=A(z)+d \phi_{1}, \quad A(z+\tau)=A(z)+d \phi_{2}$,

where

$$
\phi_{1}=\frac{\pi M}{\operatorname{Im} \tau} \operatorname{Im} z, \quad \phi_{2}=\frac{\pi M}{\operatorname{Im} \tau} \operatorname{Im} \bar{\tau} z .
$$

Now, let us study the spinor field with U(1) charge $q$ on $T^{2}$,

$$
\psi(z, \bar{z})=\left(\begin{array}{c}
\psi_{+} \\
\psi_{-}
\end{array}\right) .
$$

We use the gamma matrices,

$\Gamma^{z}=(2 \pi R)^{-1}\left(\begin{array}{ll}0 & 2 \\ 0 & 0\end{array}\right), \quad \Gamma^{\bar{z}}=(2 \pi R)^{-1}\left(\begin{array}{ll}0 & 0 \\ 2 & 0\end{array}\right)$.

Then, the Dirac operator on $\psi$ is written by

$$
i \not D=i \Gamma^{z} \nabla_{z}+i \Gamma^{\bar{z}} \nabla_{\bar{z}}=\frac{i}{\pi R}\left(\begin{array}{cc}
0 & D^{\dagger} \\
D & 0
\end{array}\right),
$$

where

$$
D^{\dagger} \equiv \partial-q \frac{\pi M}{2 \operatorname{Im} \tau} \bar{z}, \quad D \equiv \bar{\partial}+q \frac{\pi M}{2 \operatorname{Im} \tau} z .
$$

Thus, the zero mode equations of spinor are written by

$$
D \psi_{+}=0, \quad D^{\dagger} \psi_{-}=0 \text {. }
$$

Also, they must satisfy the following boundary condition:

$\psi_{ \pm}(z+1)=e^{i q \phi_{1}(z)} \psi_{ \pm}(z)=\exp \left\{i \frac{\pi q M}{\operatorname{Im} \tau} \operatorname{Im} z\right\} \psi_{ \pm}(z)$,

$\psi_{ \pm}(z+\tau)=e^{i q \phi_{2}(z)} \psi_{ \pm}(z)=\exp \left\{i \frac{\pi q M}{\operatorname{Im} \tau} \operatorname{Im} \bar{\tau} z\right\} \psi_{ \pm}(z)$

because of Eq. (6). The magnetic flux should be quantized and $q M$ must be an integer.

If $q M>0, \psi_{-}$has no zero-mode, but $\psi_{+}$has $q M$ zeromodes and their wave functions are written as

$$
\psi_{+}^{j, M}(z)=\mathcal{N} e^{i \pi q M z \frac{\mathrm{Im} z}{\mathrm{~m} \tau} \cdot} \cdot \vartheta\left[\begin{array}{c}
\frac{j}{q M} \\
0
\end{array}\right](q M z, q M \tau),
$$

with $j=0,1, \ldots,(q M-1)$, where $\vartheta$ denotes the Jacobi theta function, 


$$
\vartheta\left[\begin{array}{l}
a \\
b
\end{array}\right](\nu, \tau)=\sum_{l \in \mathbf{Z}} e^{\pi i(a+l)^{2} \tau} e^{2 \pi i(a+l)(\nu+b)} .
$$

Here, $\mathcal{N}$ denotes the normalization factor given by

$$
\mathcal{N}=\left(\frac{2 \operatorname{Im} \tau|q M|}{\mathcal{A}^{2}}\right)^{1 / 4}
$$

with $\mathcal{A}=4 \pi^{2} R^{2} \operatorname{Im} \tau$. See Appendix A for computation of $\mathcal{N}$.

If $q M<0, \psi_{+}$has no zero-mode, but $\psi_{-}$has $|q M|$ zeromodes. Their wave functions are the same as the above except replacing $q M$ by $|q M|$. Thus, introducing magnetic flux leads to a chiral theory.

For simplicity, we normalize the charge $q=1$. We can discuss other charges $q \neq 1$ by replacing $M \rightarrow q M$ in the following analysis. Hereafter, we also set $M>0$. Thus, in what follows, we consider the zero-mode wave functions,

$$
\psi^{j, M}(z, \tau)=\mathcal{N} \cdot e^{i \pi M z \operatorname{Im} z / \operatorname{Im} \tau} \cdot \vartheta\left[\begin{array}{c}
\frac{j}{M} \\
0
\end{array}\right](M z, M \tau) .
$$

Here, we write $\tau$ explicitly in $\psi^{j, M}(z, \tau)$ because $\tau$ dependence is important in the following analysis. We can use another basis of zero-mode solutions,

$$
\chi^{j, M}(\tau, z)=\frac{\mathcal{N}}{\sqrt{M}} \cdot e^{i \pi M z \operatorname{Im} z / \operatorname{Im} \tau} \cdot \vartheta\left[\begin{array}{c}
0 \\
\frac{j}{M}
\end{array}\right](z, \tau / M) .
$$

These are related with each other as

$$
\begin{aligned}
\chi^{j, M} & =\frac{1}{\sqrt{M}} \sum_{k} e^{2 \pi i \frac{i k}{M}} \psi^{k, M}, \\
\psi^{j, M} & =\frac{1}{\sqrt{M}} \sum_{k} e^{-2 \pi i \frac{j k}{M}} \chi^{k, M} .
\end{aligned}
$$

See Appendix B for these relations.

Using these wave functions, we can compute 3-point coupling [5],

$$
\int d^{2} z \psi^{j_{1}, M_{1}}(z) \psi^{j_{2}, M_{2}}(z) \psi^{j_{2}, M_{2}}(z)
$$

as well as $n$-point couplings [6],

$$
\int d^{2} z \psi^{j_{1}, M_{1}}(z) \psi^{j_{2}, M_{2}}(z) \cdots \psi^{j_{n}, M_{n}}(z) .
$$

\section{B. $T^{2} / Z_{2}$ orbifold}

In [14], the zero-mode wave functions on the $T^{2} / Z_{2}$ orbifold were studied. On the $T^{2} / Z_{2}$ orbifold, we identify $z \sim-z$. Under the $Z_{2}$ twist, the zero-mode wave functions satisfy the following simple relation:

$$
\psi^{j, M}(-z)=\psi^{M-j, M}(z) .
$$

Note that $\psi^{0, M}(z)=\psi^{M, M}(z)$. The other basis, $\chi^{j, M}(z)$, also satisfies the same relation. Thus, the $Z_{2}$ even and odd wave functions $\Theta_{ \pm 1}^{j, M}(z)$ can be written by

$$
\Theta_{ \pm 1}^{j, M}(z)=\frac{1}{\sqrt{2}}\left(\psi^{j, M}(z) \pm \psi^{M-j, M}(z)\right) .
$$

The numbers of even and odd modes are shown in Table I.

By using $Z_{2}$ eigenfunctions, $\Theta_{ \pm 1}^{j, M}(z)$, we can compute 3 -point couplings and higher order couplings similar to Eqs. (22) and (23). Then, we obtain phenomenological interesting results e.g., the realization of quark and lepton mass hierarchies and their mixing angles [20-23].

We also give a comment on Scherk-Schwarz phases and discrete Wilson lines. These degrees of freedom are equivalent to each other [28]. Hence, we restrict ourselves to Scherk-Schwarz phases. With Scherk-Schwarz phases $\left(\beta_{1}, \beta_{\tau}\right)$, the boundary conditions (13) and (14) change as

$$
\begin{aligned}
& \psi(z+1)=e^{i \phi_{1}(z)+2 \pi i \beta_{1}} \psi(z), \\
& \psi(z+\tau)=e^{i \phi_{2}(z)+2 \pi i \beta_{\tau}} \psi(z),
\end{aligned}
$$

for $q=1$. On the orbifold, discrete values of ScherkSchwarz phases are possible [28]. (See also [33].) On the $T^{2} / Z_{2}$ orbifold, there are four possible Scherk-Schwarz phases,

$\left(\beta_{1}, \beta_{\tau}\right)=(0,0), \quad(0,1 / 2), \quad(1 / 2,0), \quad(1 / 2,1 / 2)$.

For such boundary conditions, the zero-mode wave functions are obtained as [28]

$\psi^{j+\beta_{1}, \beta_{\tau}, M}(z)=\mathcal{N} \cdot e^{i \pi M z \operatorname{Im} z / \operatorname{Im} \tau} \cdot \vartheta\left[\begin{array}{c}\frac{j+\beta_{1}}{M} \\ -\beta_{\tau}\end{array}\right](M z, M \tau)$.

Under the $Z_{2}$ twist, these wave functions behave as

$$
\begin{aligned}
\psi^{j+\beta_{1}, \beta_{\tau}, M}(-z) & =\psi^{M-j-\beta_{1},-\beta_{\tau}, M}(z) \\
& =e^{-4 \pi i \frac{\left(j+\beta_{1}\right) \beta_{\tau}}{M}} \psi^{M-j-\beta_{1}, \beta_{\tau}, M}(z) .
\end{aligned}
$$

Using this behavior, we can construct $Z_{2}$ eigenstates similar to Eq. (25).

TABLE I. The numbers of $Z_{2}$ even and odd zero-modes.

\begin{tabular}{lcc}
\hline \hline $\mathrm{M}$ & $2 n$ & $2 n+1$ \\
\hline$Z_{2}$ even & $n+1$ & $n+1$ \\
$Z_{2}$ odd & $n-1$ & $n$ \\
\hline \hline
\end{tabular}




\section{III. $T^{2} / Z_{4}$ ORBIFOLD}

Here, we study $T^{2} / Z_{4}$ orbifold models.

\section{A. Modular transformation}

We denote the basis vectors of the lattice $\Lambda$ by $\left(\alpha_{1}, \alpha_{2}\right)$ to construct $T^{2}=R^{2} / \Lambda$, i.e., $\alpha_{1}=2 \pi R$ and $\alpha_{2}=2 \pi R \tau$ in the complex basis. The same lattice can be described by another basis, $\left(\alpha_{1}^{\prime}, \alpha_{2}^{\prime}\right)$, and these lattice bases are related with each other as,

$$
\left(\begin{array}{l}
\alpha_{2}^{\prime} \\
\alpha_{1}^{\prime}
\end{array}\right)=\left(\begin{array}{ll}
a & b \\
c & d
\end{array}\right)\left(\begin{array}{l}
\alpha_{2} \\
\alpha_{1}
\end{array}\right),
$$

where $a, b, c, d$ are integer with satisfying $a d-b d=1$. That is $\operatorname{SL}(2, Z)$ transformation. The lattice basis $\left(\alpha_{1}, \alpha_{2}\right)$ spans exactly the same lattice as the basis $\left(-\alpha_{1},-\alpha_{2}\right)$. Thus, the modular transformation is $S L(2, Z) / Z_{2}$.

Under the above transformation (31), the modular parameter $\tau$ transforms as

$$
\tau \rightarrow \frac{a \tau+b}{c \tau+d}
$$

This transformation includes two important generators, $S$ and $T$,

$$
\begin{gathered}
S: \tau \rightarrow-\frac{1}{\tau}, \\
T: \tau \rightarrow \tau+1 .
\end{gathered}
$$

Here, we study $S$ because it is relevant to the $Z_{4}$ twist. $S$ transforms the lattice basis as

$$
\left(\alpha_{1}, \alpha_{2}\right) \rightarrow\left(-\alpha_{2}, \alpha_{1}\right)
$$

This is nothing but the $Z_{4}$ twist, for $\tau=i$. More precisely we can refer to this as the inverse of the $Z_{4}$ twist, i.e., the $-\pi / 2$ rotation.

\section{B. $T^{2} / Z_{4}$ orbifold model}

Here, we study the transformation behavior of zeromode wave functions under $S$. Let us start with $\chi^{j, M}(z, \tau)$. Then, we examine its $S$ transformation. That is, we replace $\tau \rightarrow-1 / \tau, z \rightarrow z / \tau$ in $\chi^{j, M}(z, \tau)$. It is found that

$$
\chi^{j, M}(z / \tau,-1 / \tau)=\psi^{j, M}(z, \tau) .
$$

To show this transformation, we have used the following relation:

$$
\vartheta\left[\begin{array}{l}
0 \\
a
\end{array}\right]\left(\frac{\nu}{\kappa},-\frac{1}{\kappa}\right)=(-i \kappa)^{1 / 2} e^{i \pi \nu^{2} / \kappa} \cdot \vartheta\left[\begin{array}{l}
a \\
0
\end{array}\right](\nu, \kappa) .
$$

That is, the $\vartheta$ function in $\chi^{j, M}(z, \tau)$ transforms

$$
\vartheta\left[\begin{array}{c}
0 \\
\frac{j}{M}
\end{array}\right]\left(z, \frac{\tau}{M}\right) \rightarrow(-i M \tau)^{1 / 2} e^{i \pi M \frac{z^{2}}{\tau}} \cdot \vartheta\left[\begin{array}{c}
\frac{j}{M} \\
0
\end{array}\right](M z, M \tau) .
$$

In addition, we combine the $S$ transformation of the phase $e^{i \pi M z \frac{\mathrm{m} z}{\operatorname{lm} \tau}}$ with the phase factor $e^{i \pi M \frac{z^{2}}{\tau}}$ in the above equation (38) to find

$\exp \left\{\pi i M \frac{z}{\tau} \frac{\operatorname{Im} z / \tau}{\operatorname{Im}(-1 / \tau)}+\pi i M \frac{z^{2}}{\tau}\right\}=\exp \left\{\pi i M \frac{z \cdot \operatorname{Im} z}{\operatorname{Im} \tau}\right\}$.

Also the normalization factor transforms under $S$,

$$
\mathcal{N} \rightarrow\left(\frac{1}{|\tau|^{2}}\right)^{1 / 4} \mathcal{N}
$$

Using these results, we can derive the transformation (36) $[5] .^{2}$

On the other hand, we replace

$$
\tau \rightarrow-1 / \tau, \quad z \rightarrow \tau z,
$$

in $\psi^{j, M}(z, \tau)$. Similarly, we find that

$$
\psi^{j, M}\left(\tau z,-\frac{1}{\tau}\right)=\chi^{j, M}(z, \tau) .
$$

We require that the torus is invariant under the $S$ transformation, i.e.

$$
\tau=-\frac{1}{\tau} .
$$

Its solution is $\tau= \pm i$. Here, we set $\tau=i$. Then, the above transformation (41) is nothing but the $Z_{4}$ twist, $z \rightarrow \tau z=i z$. Thus, under such $Z_{4}$ twist, wave functions transform

$$
\begin{aligned}
\psi^{j, M}(z, \tau=i) & \rightarrow \psi^{j, M}(i z,-1 / \tau=i) \\
& =\chi^{j, M}(z, \tau=i) \\
& =C_{k, M}^{j} \psi^{k, M}(z, \tau=i) .
\end{aligned}
$$

In the last equality, we have used the relation (20), and the coefficients $C_{k, M}^{j}$ are written by

\footnotetext{
${ }^{2}$ Such a transformation behavior is important in modular symmetry of 4D low-energy effective field theory [34].
} 


$$
C_{k, M}^{j}=\frac{1}{\sqrt{M}} e^{2 \pi i \frac{j k}{M}} .
$$

The matrix $C_{k, M}^{j}$ satisfies

$$
\sum_{k} C_{k, M}^{j} C_{l, M}^{k}=\frac{1}{M} \sum_{k} e^{2 \pi i(j+l) k / M}=\delta_{(j+l), n M},
$$

where $n$ is integer. That is, we find that the $Z_{4}$ transformation,

$$
\begin{aligned}
\psi^{j, M}(z, i) & \rightarrow \chi^{j, M}(z, i) \rightarrow \psi^{M-j, M}(z, i) \\
& \rightarrow \chi^{M-j, M}(z, i) \rightarrow \psi^{j, M}(z, i) .
\end{aligned}
$$

This transformation property is consistent with the $Z_{2}$ transformation (24). That is, we can write

$$
\begin{aligned}
\psi^{j, M}(z, i) & \rightarrow \chi^{j, M}(z, i) \rightarrow \psi^{j, M}(-z, i) \\
& \rightarrow \chi^{j, M}(-z, i) \rightarrow \psi^{j, M}(z, i),
\end{aligned}
$$

and the operation of the $Z_{4}$ twist 2 times is just the $Z_{2}$ twist.

Now, we can write the zero-mode wave functions with $Z_{4}$ eigenvalues $\gamma= \pm 1, \pm i$ as

$$
\begin{aligned}
& \frac{1}{2}\left(\psi^{j, M}(z, i)+\gamma^{-1} \chi^{j, M}(z, i)+\gamma^{-2} \psi^{M-j, M}(z, i)\right. \\
& \left.\quad+\gamma^{-3} \chi^{M-j, M}(z, i)\right),
\end{aligned}
$$

i.e.,

$$
\begin{aligned}
& \frac{1}{2}\left(\psi^{j, M}(z, i)+\gamma^{-1} \sum_{k} C_{k, M}^{j} \psi^{k, M}(z, i)\right. \\
& \left.\quad+\gamma^{-2} \psi^{M-j, M}(z, i)+\gamma^{-3} \sum_{k} C_{k, M}^{M-j} \psi^{k, M}(z, i)\right) .
\end{aligned}
$$

Obviously, we can construct the $Z_{4}$ eigenstates as those of the matrix $C_{k, M}^{j}$. As an illustrating example, we study the model with $M=3$, where the matrix $C_{k, M}^{j}$ is obtained as

$$
C_{k, M}^{j}=\frac{1}{\sqrt{3}}\left(\begin{array}{ccc}
1 & 1 & 1 \\
1 & \rho & \rho^{2} \\
1 & \rho^{2} & \rho
\end{array}\right),
$$

with $\rho=2 \pi i / 3 .^{3}$ This matrix has eigenvalues, $\gamma=1,-1, i$, and eigenvectors in the basis $\sum_{j} a_{j} \psi^{j, 3}$,

\footnotetext{
${ }^{3}$ This matrix is the same as the matrix representation of $S$ in heterotic string theory on the $Z_{3}$ orbifold [35].
}

TABLE II. The number of zero-modes in the $Z_{4}$ orbifold model.

\begin{tabular}{lcccccccccccc}
\hline \hline$M$ & 1 & 2 & 3 & 4 & 5 & 6 & 7 & 8 & 9 & 10 & 11 & 12 \\
\hline$Z_{4}$ eigenvalue: +1 & 1 & 1 & 1 & 2 & 2 & 2 & 2 & 3 & 3 & 3 & 3 & 4 \\
$Z_{4}$ eigenvalue: -1 & 0 & 1 & 1 & 1 & 1 & 2 & 2 & 2 & 2 & 3 & 3 & 3 \\
$Z_{4}$ eigenvalue: $+i$ & 0 & 0 & 1 & 1 & 1 & 1 & 2 & 2 & 2 & 2 & 3 & 3 \\
$Z_{4}$ eigenvalue: $-i$ & 0 & 0 & 0 & 0 & 1 & 1 & 1 & 1 & 2 & 2 & 2 & 2 \\
\hline \hline
\end{tabular}

TABLE III. Generic results on the numbers of $Z_{4}$ zero-modes.

\begin{tabular}{lcccc}
\hline \hline$M$ & $4 n$ & $4 n+1$ & $4 n+2$ & $4 n+3$ \\
\hline$Z_{4}$ eigenvalue: +1 & $n+1$ & $n+1$ & $n+1$ & $n+1$ \\
$Z_{4}$ eigenvalue: -1 & $n$ & $n$ & $n+1$ & $n+1$ \\
$Z_{4}$ eigenvalue: $+i$ & $n$ & $n$ & $n$ & $n+1$ \\
$Z_{4}$ eigenvalue: $-i$ & $n-1$ & $n$ & $n$ & $n$ \\
\hline \hline
\end{tabular}

$$
\begin{aligned}
& \left(a_{0}, a_{1}, a_{2}\right)=(1+\sqrt{3}, 1,1) \text { for } \gamma=1, \\
& (1-\sqrt{3}, 1,1) \text { for } \gamma=-1, \\
& (0,1,-1) \text { for } \gamma=i,
\end{aligned}
$$

up to normalization factors.

Similarly, we can obtain $Z_{4}$ eigenvalues and eigenstates by using explicit matrices, $C_{k, M}^{j}$ for each value of $M$, in particular small values of $M$. Table II shows the numbers of $Z_{4}$ zero-modes for small values of $M$. This result is consistent with the previous results $[28,29]$ up to the definition of the $Z_{4}$ twist. $^{4}$ The corresponding $Z_{4}$ eigenstates are shown in Appendix C.

We give a comment on $Z_{4}$ eigenstates. The $Z_{2}$ even states, $\left(\psi^{j, M}+\psi^{M-j, M}\right)$, correspond to the $Z_{4}$ eigenstates with eigenvalues $\gamma= \pm 1$, while $Z_{2}$ odd states, $\left(\psi^{j, M}-\psi^{M-j, M}\right)$, correspond to the $Z_{4}$ states with eigenvalues $\gamma= \pm i$. Explicit results on eigenstates for small number of $M$ are shown in Appendix C. For $M=$ even, $Z_{4}$ eigenvectors are relatively simple, while for $M=$ odd $Z_{4}$ eigenvectors are complicated.

From the above explicit results, we can expect generic results on the numbers of zero-modes, which are shown in Table III. Indeed, we can prove this result. First, we compute $\operatorname{tr} C_{k, M}^{j}$,

$$
\operatorname{tr} C=\frac{1}{\sqrt{M}} \sum_{k=0}^{M-1} e^{2 \pi i \frac{k^{2}}{M}}
$$

In our computation, the following Landsberg-Schaar relation:

\footnotetext{
${ }^{4}$ The number of zero-modes with $Z_{4}$ eigenvalue $\gamma=i$ is exchanged for the number of zero-modes with eigenvalue $\gamma=-i$ when we replace the definition of $Z_{4}$ twist by its inverse.
} 


$$
\frac{1}{\sqrt{p}} \sum_{n=0}^{p-1} e^{\frac{2 \pi i n^{2} q}{p}}=\frac{e^{\frac{\pi i}{4}}}{\sqrt{2 q}} \sum_{n=0}^{2 q-1} e^{-\frac{\pi n^{2} p}{2 q}}, \quad p, q \in \mathbb{N},
$$

is very useful. We take $p=M, q=1$ in the LandsbergSchaar relation to compute $\operatorname{tr} C$,

$$
\operatorname{tr} C=e^{\frac{\pi i}{4}} \frac{1}{\sqrt{2}} \sum_{k=0}^{1} e^{-\frac{\pi i k^{2} M}{2}}=e^{\frac{\pi i}{4}} \frac{1}{\sqrt{2}}\left(1+e^{-\pi i \frac{M}{2}}\right) .
$$

Then, we find that

$$
\operatorname{tr} C= \begin{cases}1+i & \text { for } M=4 n \\ 1 & \text { for } M=4 n+1 \\ 0 & \text { for } M=4 n+2 \\ i & \text { for } M=4 n+3\end{cases}
$$

For example, recall that when $M=4 n$, there are $(2 n+1) Z_{2}$ even zero-modes and $(2 n-1) Z_{2}$ odd zeromodes. That is, the sum of the numbers of $Z_{4}$ zero-modes with eigenvalues $\gamma= \pm 1$ is equal to $(2 n+1)$, while the sum of the numbers of $Z_{4}$ zero-modes with eigenvalues $\gamma= \pm i$ is equal to $(2 n-1)$. Combination of these with Eq. (56) leads to the result for $M=4 n$ in Table III. Similarly, we can derive the numbers of $Z_{4}$ zero-modes with other values of $M$ as shown in Table III.

\section{IV. $T^{2} / Z_{3}$ ORBIFOLD}

In this section, we study the zero-modes on $T^{2} / Z_{3}$ and $T^{2} / Z_{6}$ orbifolds.

\section{A. $T^{2} / Z_{3}$ orbifold}

Here, we study the $Z_{3}$ orbifold models. Our strategy is the same as one in the previous section. That is, we examine the modular transformation corresponding to the $Z_{3}$ twist. A good candidate for the $Z_{3}$ twist is $S T$ transformation, because it satisfies $(S T)^{3}=1$ on $\tau$. Under $S T$, the modular parameter $\tau$ transforms as

$$
\tau \rightarrow-\frac{1}{\tau+1}
$$

When $\tau=e^{ \pm 2 \pi i / 3}$, the modular parameter is invariant under $S T$, i.e.

$$
\tau=-\frac{1}{\tau+1} .
$$

For such a transformation, the $Z_{3}$ twist (its inverse) can be defined by

$$
z \rightarrow \tau z
$$

when $\tau=e^{2 \pi i / 3}\left(\tau=e^{-2 \pi i / 3}\right)$. Alternatively, we can define the $Z_{3}$ twist by

$$
z \rightarrow \frac{-z}{\tau+1}
$$

because of the relation (58). In what follows, we study the transformation of wave functions under Eqs. (57) and (60). We restrict ourselves to the models with $M=$ even, because the following transformation behavior is valid only for $M=$ even.

We find that

$$
\begin{aligned}
\chi^{j, M}(-z /(\tau+1),-1 /(\tau+1)) & =e^{\pi i i^{2^{2}}} \cdot \psi^{j, M}(-z, \tau) \\
& =e^{\pi i \frac{i^{2}}{M}} \cdot \psi^{M-j, M}(z, \tau) .
\end{aligned}
$$

Here, we have used the relation (37) and the following relation:

$\theta\left[\begin{array}{l}a \\ b\end{array}\right](\nu, \tau+1)=e^{-i \pi a(a-1)} \cdot \theta\left[\begin{array}{c}a \\ b+a-\frac{1}{2}\end{array}\right](\nu, \tau)$.

Since $\psi^{M-j, M}=C_{j k} \chi^{k, M}$, the transformation in the $\chi$ basis is written by

$\chi^{j, M}(z, \tau) \rightarrow D_{k, M}^{j} \chi^{k, M}(z, \tau), \quad D_{k, M}^{j}=e^{\pi i_{M}^{2}} C_{k, M}^{j}$.

When we examine the inverse transformation,

$$
\tau \rightarrow-\frac{1}{\tau}-1, \quad z \rightarrow \frac{1}{\tau} z,
$$

on the wave function $\psi^{j, M}(z, \tau)$, we find that

$$
\psi^{j, M}\left(\frac{z}{\tau},-1 / \tau-1\right)=e^{-\pi i \frac{j^{2}}{M}} \cdot \chi^{M-j}(z, \tau) .
$$

Thus, it is found that under the above inverse transformation, the wave function $\psi^{j, M}$ transforms as

$$
\psi^{j, M} \rightarrow\left(D^{-1}\right)_{k, M}^{j} \psi^{j, M},
$$

where $D^{-1}$ is the inverse matrix of $D_{k, M}^{j}$.

For example, for $M=2$, we obtain

$$
D_{k, M=2}^{j}=\frac{1}{\sqrt{2}}\left(\begin{array}{cc}
1 & 1 \\
i & -i
\end{array}\right) .
$$

However, we find that

$$
\left(D_{k, M=2}^{j}\right)^{3}=\frac{1}{\sqrt{2}}\left(\begin{array}{cc}
1+i & 0 \\
0 & 1+i
\end{array}\right) .
$$

This matrix does not realize exactly the $Z_{3}$ twist. 
Indeed, for generic even number $M$, we can find

$$
D_{k, M}^{j} D_{\ell, M}^{k} D_{m, M}^{\ell}=\frac{1}{\sqrt{2}}(1+i) \delta_{j, m}=e^{\pi i / 4} \delta_{j, m} .
$$

See Appendix B. Thus, the matrix $D_{k, M}^{j}$ on $\chi^{j, M}$ does not represent the $Z_{3}$ twist exactly.

Here, we allow the constant phase for all modes under the above transformation, e.g. ${ }^{5}$

$\chi^{j, M}(z, \tau) \rightarrow \tilde{D}_{k, M}^{j} \chi^{k, M}(z, \tau), \quad \tilde{D}_{k, M}^{j}=e^{-\frac{\pi i}{12}} D_{k, M}^{j}$.

Then, we can realize the $Z_{3}$ twist,

$$
\tilde{D}_{k, M}^{j} \tilde{D}_{\ell, M}^{k} \tilde{D}_{m, M}^{\ell}=\delta_{j, m} .
$$

Here, we employ this matrix $\tilde{D}$ as the $Z_{3}$ twist.

For example, for $M=2$, we use the following matrix for the $Z_{3}$ twist on $\chi^{j, M}$ :

$$
\tilde{D}_{k, M=2}^{j}=\frac{e^{-\frac{\pi i}{12}}}{\sqrt{2}}\left(\begin{array}{cc}
1 & 1 \\
i & -i
\end{array}\right) .
$$

TABLE IV. The number of zero-modes in the $Z_{3}$ orbifold model.

\begin{tabular}{lcccccc}
\hline \hline$M$ & 2 & 4 & 6 & 8 & 10 & 12 \\
\hline$Z_{3}$ eigenvalue: 1 & 1 & 1 & 3 & 3 & 3 & 5 \\
$Z_{3}$ eigenvalue: $e^{2 \pi i / 3}$ & 0 & 2 & 2 & 2 & 4 & 4 \\
$Z_{3}$ eigenvalue: $e^{-2 \pi i / 3}$ & 1 & 1 & 1 & 3 & 3 & 3 \\
\hline \hline
\end{tabular}

Its eigenvalues are obtained as $\gamma=1, e^{-2 \pi i / 3}$, and eigenvectors are given as

$$
\left(1, \sqrt{2} \gamma e^{\frac{\pi i}{12}}-1\right)
$$

in the $\left(\chi^{0, M}, \chi^{1, M}\right)$ basis, up to a normalization factor.

Similarly, we study the model with $M=4$. The eigenvalues of the matrix $\tilde{D}_{k, M}^{j}$ are

$$
\left(1, e^{2 \pi i / 3}, e^{2 \pi i / 3}, e^{-2 \pi i / 3}\right),
$$

and their eigenvectors are obtained in the basis $a_{i} \chi_{i}$,

$$
\begin{aligned}
& {[0,-1,0,1],} \\
& {[-i(-1+\sqrt{2}),(1+i)-\sqrt{2}, 1,0],} \\
& {\left[\frac{(-6+6 i)+3 i \sqrt{2}-(2+2 i) \sqrt{3}+(1-2 i) \sqrt{6}}{3 i \sqrt{2}+(2-2 i) \sqrt{3}}, 1, \frac{\sqrt{2}(3 i+(1+2 i) \sqrt{3})}{3 i \sqrt{2}+(2-2 i) \sqrt{3}+\sqrt{6}}, 1\right],} \\
& {\left[\frac{6 i+3(-1)^{1 / 4}+(1+3 i) \sqrt{3 / 2}+2 \sqrt{3}}{3(-1)^{1 / 4}-(1-i) \sqrt{3 / 2}+2 i \sqrt{3}}, 1, \frac{\sqrt{2}(-3 i+(1+2 i) \sqrt{3}}{-3 i \sqrt{2}+(2-2 i) \sqrt{3}+\sqrt{6}}, 1\right],}
\end{aligned}
$$

up to normalization factors.

Similarly, we can analyze the eigenvalues and eigenvectors for other $M$. Table IV shows the numbers of $Z_{3}$ zero-modes with each eigenvalue for small values of $M$. This result is consistent with the previous results $[28,29]$. We can derive eigenvectors, but their explicit forms are, in general, very complicated.

We can analyze the number of $Z_{3}$ zero-modes for generic even number $M$. First we compute the trace of the inverse of $\tilde{D}$,

\footnotetext{
${ }^{5}$ We have other two values for candidates of the constant phase, and totally there are three possibilities. Different constant phases lead to a change of degeneracy factors for each $Z_{3}$ eigenvalues. Such possibilities of constant phases may correspond to the possibility of the introduction of Scherk-Schwarz phases. Similarly, we have the degree of freedom to define the $Z_{4}$ twist by $e^{\pi i n / 2} C_{k, M}^{j}$ with $n=0,1,2,3$.
}

$$
\left(\tilde{D}^{-1}\right)_{k}^{j}=e^{\pi i \frac{1}{12}} \cdot e^{-\pi i \frac{j^{2}}{M}} \cdot C_{j k}^{\dagger}
$$

That is, its trace is written by

$\operatorname{tr}\left(\tilde{D}^{-1}\right)=e^{\pi i \frac{1}{12}} \sum_{k=0}^{M-1} e^{-\pi i \frac{k^{2}}{M}} \cdot e^{-2 \pi i \frac{i^{2}}{M}}=e^{\pi i \frac{1}{12}} \sum_{k=0}^{M-1} e^{-3 \pi i \frac{k^{2}}{M}}$

Here, we use the Landsberg-Schaar relation (54) with $p=3$ and $2 q=M$. Then, we find

$$
\operatorname{tr}\left(\tilde{D}^{-1}\right)=\frac{i e^{-\pi i \frac{2}{3}}}{\sqrt{3}}\left(1+2 e^{\pi i \frac{M}{3}}\right) .
$$

Explicitly, we obtain the following results:

$$
\operatorname{tr} \tilde{D}^{-1}= \begin{cases}1+\omega & \text { for } M=6 n+2 \\ \omega^{2} & \text { for } M=6 n+4, \\ 2+\omega^{2} & \text { for } M=6 n\end{cases}
$$


TABLE V. Generic results on $Z_{3}$ zero-modes.

\begin{tabular}{lccc}
\hline \hline$M$ & $6 n$ & $6 n+2$ & $6 n+4$ \\
\hline$Z_{3}$ eigenvalue: 1 & $2 n+1$ & $2 n+1$ & $2 n+1$ \\
$Z_{3}$ eigenvalue: $e^{2 \pi i / 3}$ & $2 n$ & $2 n$ & $2 n+2$ \\
$Z_{3}$ eigenvalue: $e^{2 \pi i / 3}$ & $2 n-1$ & $2 n+1$ & $2 n+1$ \\
\hline \hline
\end{tabular}

where $\omega=e^{2 \pi i / 3}$. Then, the trace of its inverse can be obtained by replacing $\omega \rightarrow \omega^{2}$,

$$
\operatorname{tr} \tilde{D}= \begin{cases}1+\omega^{2} & \text { for } M=6 n+2 \\ \omega & \text { for } M=6 n+4 \\ 2+\omega & \text { for } M=6 n\end{cases}
$$

From this result, we can derive the number of $Z_{3}$ eigenstates as shown in Table V. Note that $1+\omega+\omega^{2}=0$.

\section{B. $Z_{6}$ orbifold}

Obviously, the $Z_{6}$ twist can realized by the product of the $Z_{2}$ and $Z_{3}$ twists. Also, recall that the $Z_{2}$ twist on $\psi^{j, M}(z)$ and $\chi^{j, M}(z)$ is realized by

$$
\begin{aligned}
\psi^{j, M}(z) & \rightarrow \psi^{j, M}(-z)=\psi^{M-j, M}(z), \\
\chi^{j, M}(z) \rightarrow \chi^{j, M}(-z) & =\chi^{M-j, M}(z) .
\end{aligned}
$$

Here, we restrict ourselves to the models with $M=$ even. From the analysis on the $T^{2} / Z_{3}$ orbifold, the $Z_{6}$ twist can be realized by

$$
F_{k, M}^{j}=e^{\frac{\pi i}{12}} e^{-\pi i i_{M}^{2}} C_{k, M}^{j} .
$$

Again, using the Landsberg-Schaar relation (54), we compute the trace of $F_{k, M}^{j}$ matrix,

$$
\operatorname{tr} F=e^{\frac{\pi i}{12}} \sum_{k} e^{\pi i \frac{k^{2}}{M}}=e^{\frac{\pi i}{12}} e^{\frac{\pi i}{4}}
$$

The possible eigenvalues of $F$-matrix are $\gamma=\rho^{k}$ with $k=0,1, \ldots, 5$ and $\rho=e^{\pi i / 3}$. Here, we denote the number of zero-modes with eigenvalues $\gamma$ by $N_{\gamma}$. Since $\left(F_{k, M}^{j}\right)^{3}$ corresponds to the $Z_{2}$ twist, the zero-mode numbers, $N_{\gamma}$ must satisfy

$N_{1}+N_{\rho^{2}}+N_{\rho^{4}}=n+1, \quad N_{\rho}+N_{\rho^{3}}+M_{\rho^{5}}=n-1$,

for $M=2 n$. Similarly, $\left(F_{k, M}^{j}\right)^{2}$ corresponds to the $Z_{3}$ twist, the zero-mode numbers must satisfy

$$
N_{1}+N_{\rho^{3}}=2 n+1,
$$

TABLE VI. Generic results on $Z_{6}$ zero-modes.

\begin{tabular}{lccc}
\hline \hline$M$ & $6 n$ & $6 n+2$ & $6 n+4$ \\
\hline eigenvalue: 1 & $n+1$ & $n+1$ & $n+1$ \\
eigenvalue: $e^{\pi i / 3}$ & $n$ & $n$ & $n+1$ \\
eigenvalue: $e^{2 \pi i / 3}$ & $n$ & $n+1$ & $n+1$ \\
eigenvalue: $e^{3 \pi i / 3}$ & $n$ & $n$ & $n$ \\
eigenvalue: $e^{4 \pi i / 3}$ & $n$ & $n$ & $n+1$ \\
eigenvalue: $e^{5 \pi i / 3}$ & $n-1$ & $n$ & $n$ \\
\hline \hline
\end{tabular}

for $M=6 n, 6 n+2,6 n+4$,

$$
N_{\rho}+N_{\rho^{4}}=\left\{\begin{array}{ll}
2 n & \text { for } M=6 n, 6 n+2 \\
2 n+2 & \text { for } M=6 n+4
\end{array},\right.
$$

and

$N_{\rho^{2}}+N_{\rho^{5}}=\left\{\begin{array}{ll}2 n-1 & \text { for } M=6 n \\ 2 n+1 & \text { for } M=6 n+2,6 n+4\end{array}\right.$.

Combining these relations with the trace (83), we find the number of eigenstates, which is shown in Table VI.

In principle, we can derive zero-mode wave functions with eigenvalues $\gamma$, but its explicit form is complicated.

\section{ZERO-MODES ON ORBIFOLDS}

We have studied the zero-modes on several orbifolds, $T^{2} / Z_{N}$ with $N=2,3,4,6$. Now, let us compare our results between different $T^{2} / Z_{N}$ orbifolds. We examine the $Z_{N}$ invariant zero-modes. It is found that the number of $Z_{N}$ invariant zero-modes is written by

$$
I_{M, N}=\lfloor M / N\rfloor+1,
$$

on $T^{2} / Z_{N}$ orbifold with magnetic flux $M$ except the $Z_{3}$ orbifold with $M=6 n+4$. Here, $\lfloor r\rfloor$ denotes the maximum integer $n$, which satisfies $n \leq r$. Alternatively, the number of $Z_{3}$ invariant zero-modes is written by

$$
I_{M, N}^{(3)}=2\lfloor M /(2 N)\rfloor+1 .
$$

Our results are quite universal for different $T^{2} / Z_{N}$ orbifolds.

The index theorem tells that the number of zero-modes of the Dirac operators on $T^{2}$ with flux $M$ is equal to $M$. The above number $I_{M, N}$ as well as $I_{M, N}^{(3)}$ would correspond to such an index on the $T^{2} / Z_{N}$ orbifolds.

It would be useful to rewrite the numbers of zero-modes with other eigenvalues by using the symbol $\lfloor r\rfloor$. These are shown in Table VII. Note that the number of zero-modes with $Z_{N}$ eigenvalue $\gamma$ is exchanged for one with $Z_{N}$ eigenvalue $\gamma^{-1}$ when we replace the definition of $Z_{N}$ twist by its inverse. 
TABLE VII. Generic results on $Z_{N}$ zero-modes.

\begin{tabular}{lc}
\hline \hline Eigenvalues $(\gamma)$ & Number of zero-modes \\
\hline$Z_{N=2 n}$ invariant & $\lfloor M / N\rfloor+1$ \\
$Z_{3}$ invariant & $2\lfloor M /(2 N)\rfloor+1$ \\
$Z_{2}(\gamma=-1)$ & $\lfloor(M-1) / 2\rfloor$ \\
$Z_{4}(\gamma=-1)$ & $\lfloor(M-2) / 4\rfloor+1$ \\
$Z_{4}(\gamma=i)$ & $\lfloor(M-3) / 4\rfloor+1$ \\
$Z_{4}(\gamma=-i)$ & $\lfloor(M-1) / 4\rfloor$ \\
$Z_{3}\left(\gamma=e^{2 \pi i / 3}\right)$ & $2\lfloor(M-4) / 6\rfloor+2$ \\
$Z_{3}\left(\gamma=e^{-2 \pi i / 3}\right)$ & $2\lfloor(M-2) / 6\rfloor+1$ \\
$Z_{6}\left(\gamma=e^{\pi i / 3}\right)$ & $\lfloor(M-4) / 6\rfloor+1$ \\
$Z_{6}\left(\gamma=e^{2 \pi i / 3}\right)$ & $\lfloor(M-2) / 6\rfloor+1$ \\
$Z_{6}\left(\gamma=e^{3 \pi i / 3}\right)$ & $\lfloor M / 6\rfloor$ \\
$Z_{6}\left(\gamma=e^{4 \pi i / 3}\right)$ & $\lfloor(M-4) / 6\rfloor+1$ \\
$Z_{6}\left(\gamma=e^{5 \pi i / 3}\right)$ & $\lfloor(M-2) / 6\rfloor$ \\
\hline \hline
\end{tabular}

It seems that the $T^{2} / Z_{3}$ orbifold has the zero-mode structure different from the other orbifolds. The numbers of zero-modes on $T^{2} / Z_{N}$ with $N=$ even have the structure with the period $N$ for $M$. That is, the number of zero-modes increases by one when we replace $M$ by $M+N$. On the other hand, the number of zero-modes on $T^{2} / Z_{3}$ has the structure with the period 6 , and the number of zero-modes increases by 2 when replace $M$ by $M+6$. Such a structure of $T^{2} / Z_{3}$ is similar to one of $T^{2} / Z_{6}$ and seems to be originated from the $T^{2} / Z_{6}$ orbifold. At any rate, the deep reason why the $T^{2} / Z_{3}$ orbifold has a different structure is not clear. It is important to study its reason further.

The number of $Z_{N}$ invariant zero-modes depends on nontrivial Scherk-Schwarz phases and discrete Wilson lines. Thus, our results imply that the number of $Z_{N}$ invariant zero-modes is universal over all of $T^{2} / Z_{N}$ orbifolds if we choose proper conditions on Scherk-Schwarz phases and discrete Wilson lines.

\section{CONCLUSION}

We have studied $T^{2} / Z_{N}$ orbifold models with magnetic flux. We used the modular transformation to define the orbifolds. Then, we have computed zero-mode wave functions with each eigenvalue of the $Z_{N}$ twist. We have shown the zero-mode numbers. It is found that the number of the $Z_{N}$ invariant zero-modes is universal among different $T^{2} / Z_{N}$ orbifolds, and it can be obtained by $\lfloor M / N\rfloor+1$ except one case in the $T^{2} / Z_{3}$ orbifold. The zero-mode number of the Dirac operator on $T^{2}$ is given by $M$. Our result would correspond to such an index.

We can write wave functions analytically for fixed $M$. Thus, we can compute 3-point couplings and higher order couplings. Hence, our results would be useful to further phenomenological applications. One can also apply our method to not only zero-modes, but also higher modes.

\section{ACKNOWLEDGMENTS}

T. K. was supported by MEXT KAKENHI Grant No. JP17H05395 and JSPS KAKENSHI Grant No. JP26247042.

\section{APPENDIX A: NORMALIZATION OF WAVE FUNCTION AND INNER PRODUCT OF $\psi$ AND $\chi$}

In this appendix, we show computations on normalization $\mathcal{N}$ of wave functions and the relations (20) and (21). The computation on normalization is useful for computation of the relations (20) and (21). Now, we compute

$$
\int_{T^{2}} d z d \bar{z} \psi^{j}\left(\psi^{k}\right)^{*}
$$

where

$$
\int_{T^{2}} d z d \bar{z}=\mathcal{A} \int_{0}^{1} d(\operatorname{Re} z) \int_{0}^{1} d\left(\frac{\operatorname{Im} z}{\operatorname{Im} \tau}\right)
$$

with $\mathcal{A}=4 \pi^{2} R^{2} \operatorname{Im} \tau$. The product of the wave functions, $\psi^{j}\left(\psi^{k}\right)^{*}$ is written explicitly,

$$
\begin{aligned}
\psi_{ \pm}^{j}\left(\psi_{ \pm}^{k}\right)^{*} & =\psi^{j, q M}(\tau, z) \cdot \psi^{-k,-q M}(\bar{\tau}, \bar{z}) \\
& =\mathcal{N}^{2} \cdot e^{-2 \pi q M(\operatorname{Im} z)^{2} / \operatorname{Im} \tau} \cdot \vartheta\left[\begin{array}{c}
\frac{j}{q M} \\
0
\end{array}\right](q M z, q M \tau) \cdot \vartheta\left[\begin{array}{c}
\frac{k}{q M} \\
0
\end{array}\right](-q M \bar{z},-q M \bar{\tau}) .
\end{aligned}
$$

The product of theta functions includes the following terms depending on $\operatorname{Re} z$ and $\operatorname{Im} z$ :

$$
\sum_{n} \sum_{n^{\prime}} e^{2 \pi i\left\{\left(\frac{j}{q M}+n\right)-\left(\frac{k}{q M}+n^{\prime}\right)\right\} \operatorname{Re} z} \cdot e^{-2 \pi\left\{\left(\frac{j}{q M}+n\right)+\left(\frac{k}{q M}+n^{\prime}\right)\right\} \operatorname{Im} z} .
$$

Then, the integration over $\operatorname{Re} z$ leads to the Kronecker delta, $\delta_{j /(q M)+n, k /(q M)+n^{\prime}}$. Thus, we obtain 


$$
\int_{0}^{1} d(\operatorname{Re} z) \psi^{j, q M}(\tau, z) \cdot \psi^{-k,-q M}(\bar{\tau}, \bar{z})=\mathcal{N}^{2} \sum_{n} e^{-2 \pi q M \operatorname{Im} \tau\left(n+\frac{j}{q M}+\frac{\operatorname{Im} z}{\operatorname{Im} \tau}\right)^{2}} .
$$

Furthermore, we can find

$$
\begin{aligned}
\int_{0}^{1} d\left(\frac{\operatorname{Im} z}{\operatorname{Im} \tau}\right) \sum_{n} e^{-2 \pi q M \operatorname{Im} \tau\left(n+\frac{j}{q M}+\frac{\operatorname{Im} z}{\operatorname{Im} \tau}\right)^{2}} & =\sum_{n} \int_{0}^{1} d\left(\frac{\operatorname{Im} z}{\operatorname{Im} \tau}\right) e^{-2 \pi q M \operatorname{Im} \tau\left(n+\frac{j}{q M}+\frac{\operatorname{Im} z}{\operatorname{Im} \tau}\right)^{2}} \\
& =\int_{-\infty}^{\infty} d x e^{-2 \pi q M \operatorname{Im} \tau x^{2}} \\
& =\left(\frac{1}{2 q M \operatorname{Im} \tau}\right)^{\frac{1}{2}}
\end{aligned}
$$

Then, we find the normalization factor (17).

Similarly, we compute

$$
\int d z d \bar{z} \chi^{j, M}(z, \tau) \cdot\left(\psi^{k, M}(z, \tau)\right)^{*}
$$

where

$$
\chi^{j, M} \cdot \psi^{-k,-M}=\frac{(\mathcal{N})^{2}}{\sqrt{M}} \cdot e^{-2 \pi M \frac{(\mathrm{Im} z)^{2}}{\operatorname{Im} \tau}} \cdot \theta\left[\begin{array}{c}
0 \\
\frac{j}{M}
\end{array}\right]\left(z, \frac{\tau}{M}\right) \cdot \theta\left[\begin{array}{c}
\frac{k}{M} \\
0
\end{array}\right](-\bar{z} M,-\bar{\tau} M) .
$$

The product of the theta functions includes the following terms depending on $\operatorname{Re} z$ :

$$
\sum_{l} \sum_{l^{\prime}} e^{2 \pi i\left\{l-M\left(l^{\prime}+\frac{k}{M}\right)\right\} \operatorname{Re} z}
$$

The integration over $\operatorname{Re} z$ leads to the Kronecker delta, $\delta_{\ell, M \ell^{\prime}+k}$. Thus, we obtain

$$
\begin{aligned}
& \int_{0}^{1} \operatorname{Re}(z) \chi^{j, M} \cdot \psi^{-k,-M} \\
& \quad=e^{2 \pi i \frac{j k}{M}} \cdot \mathcal{N}^{2} \cdot e^{-2 \pi M \frac{(\operatorname{Im} z)^{2}}{\operatorname{Im} \tau}} \sum_{l} e^{-2 \pi M \operatorname{Im} \tau\left(l+\frac{k}{M}\right)} \cdot e^{-4 \pi M\left(l+\frac{k}{M}\right) \operatorname{Im} z}
\end{aligned}
$$

In addition, we can integrate this over $\operatorname{Im}(z)$ similar to Eq. (A6). Then, we can derive

$$
\int d z d \bar{z} \chi^{j, M}(z, \tau) \cdot\left(\psi^{k, M}(z, \tau)\right)^{*}=e^{2 \pi i \frac{j k}{M}}
$$

That is nothing but the relation (20).

Also we can obtain the complex conjugate of Eq. (A11),

$$
\int d z d \bar{z} \psi^{j, M}(z, \tau) \cdot\left(\chi^{k, M}(z, \tau)\right)^{*}=e^{-2 \pi i \frac{j k}{M}},
$$

and this is nothing but the relation (21).

\section{APPENDIX B: COMPUTATION OF $(D)^{3}$}

In this section, we give the computation on $\left(D_{k, M}^{j}\right)^{3}$ for a generic even number $M$. First, we can obtain

$$
\begin{aligned}
D_{k, M}^{j} D_{\ell, M}^{k} & =\frac{1}{M} \sum_{k} e^{\frac{2 \pi i}{M}\left(\frac{l^{2}}{2}+k(j+\ell)+\frac{k^{2}}{2}\right)} \\
& =\frac{1}{M} \sum_{k} e^{\frac{\pi i}{M}\left[(k+j+\ell)^{2}-\ell(2 j+\ell)\right]} \\
& =\frac{1}{\sqrt{2 M}}(1+i) e^{\frac{\pi i}{M}[-\ell(2 j+\ell)]} .
\end{aligned}
$$

We have used the Landsberg-Schaar relation (54). Then, we can compute

$$
\begin{aligned}
D_{k, M}^{j} D_{\ell, M}^{k} D_{m, M}^{\ell} & =\frac{1}{\sqrt{2} M}(1+i) \sum_{\ell} e^{\frac{\pi i}{M}\left[-\ell(2 j+\ell)+\ell^{2}+2 \ell m\right]} \\
& =\frac{1}{\sqrt{2} M}(1+i) \sum_{\ell} e^{\frac{2 \pi i}{M} \ell(m-j)} \\
& =\frac{1}{\sqrt{2}}(1+i) \delta_{j, m}=e^{\pi i / 4} \delta_{j, m}
\end{aligned}
$$

Again, we have used the Landsberg-Schaar relation (54). 


\section{APPENDIX C: EIGENVECTORS IN $Z_{4}$ ORBIFOLD MODELS}

In this section, we give explicitly $Z_{4}$ eigenvectors for $M=2, \ldots, 12$. These eigenvectors are represented in the basis $\sum_{k=0}^{M-1} a_{k} \psi^{k, M}$. The $Z_{2}$ even states, $\left(\psi^{j, M}+\psi^{M-j, M}\right)$, correspond to the $Z_{4}$ eigenstates with eigenvalues $\gamma= \pm 1$, while $Z_{2}$ odd states, $\left(\psi^{j, M}-\psi^{M-j, M}\right)$, correspond to the $Z_{4}$ states with eigenvalues $\gamma= \pm i$. Thus, $\psi^{0, M}$ does not correspond to eigenstates with $Z_{4}$ eigenvalues $\gamma= \pm i$, but always appears as eigenstates with $Z_{4}$ eigenvalues $\gamma= \pm 1$. Similarly, when $M$ is even, $\psi^{M / 2, M}$ corresponds to only $Z_{4}$ eigenvalues $\gamma= \pm 1$. The other modes appear in all of the eigenstates with eigenvalues $\gamma= \pm 1, \pm i$. For all the cases with $M=4 n, 4 n+1,4 n+2,4 n+3$, there are $(n+1)$ independent eigenstates with $\gamma=1$. It seems convenient to use the basis such that only one of $a_{1}, a_{2}, \cdots a_{n+1}$ is nonvanishing in $\sum_{k=0}^{M-1} a_{k} \psi^{k, M}$, that is,

$$
\begin{aligned}
& \left(a_{0}, 1,0, \ldots, 0,0, a_{n+1}, a_{n+2}, \ldots, a_{M-1}\right), \\
& \left(a_{0}, 0,1,0, \ldots, 0, a_{n+1}, a_{n+2}, \ldots, a_{M-1}\right), \\
& \quad \ldots \ldots . \\
& \left(a_{0}, 0, \ldots, 0,1, a_{n+1}, a_{n+2}, \ldots, a_{M-1}\right),
\end{aligned}
$$

where the other coefficients, $a_{0}$ and $a_{n+2}, \ldots, a_{M-1}$ are determined by eigenvector equations.

Similarly, when there are $m$ independent modes, it seems convenient to use the basis such that only one of $a_{1}, a_{2}, \cdots a_{m}$ is nonvanishing. The fluxes can be classified as $M=4 n, 4 n+1,4 n+2,4 n+3$. For such classes, we show explicitly eigenvectors in the basis $\sum_{k=0}^{M-1} a_{k} \psi^{k, M}$ in what follows. As said above, the coefficients $a_{k}$ other than $a_{1}, a_{2}, \cdots a_{m}$ can be written by linear combinations of $a_{1}, a_{2}, \cdots a_{m}$. The eigenvectors for $M=$ even are relatively simple, while some of eigenvectors for $M=$ odd are written by lengthy linear combinations. In such cases, we omit writing them explicitly and just denote $L P_{i}\left(a_{1}, a_{2}, \cdots a_{m}\right)$. At any rate, $L P_{i}\left(a_{1}, a_{2}, \cdots a_{m}\right)$ can be computed by use of eigenvector equations.

\section{1. $M=4 n+2, n \in \mathbb{Z}$}

eigenvalue: +1

$M=2$

$\left(a_{0}, a_{1},\right) \propto\left((\sqrt{2}+1) a_{1}, a_{1}\right)$,

$M=6$

$$
\left(a_{0}, a_{1}, a_{2}, a_{3}, a_{4}, a_{5}\right) \propto\left(\frac{1}{2}\left(\sqrt{6} a_{1}+(2+\sqrt{6}) a_{2}\right), a_{1}, a_{2}, \frac{1}{2}\left((2-\sqrt{6}) a_{1}+\sqrt{6} a_{2}\right), a_{2}, a_{1}\right),
$$

$M=10$

$$
\begin{aligned}
& \left(a_{0}, \ldots, a_{9}\right) \propto\left(\frac{1}{2}\left((1-\sqrt{2}-\sqrt{5}+\sqrt{10}) a_{1}+(2+\sqrt{10}) a_{2}+(-1+\sqrt{2}+\sqrt{5}) a_{3}\right),\right. \\
& a_{1}, a_{2}, a_{3}, \frac{1}{2+\sqrt{10}}\left((-2+2 \sqrt{2}+2 \sqrt{5}-\sqrt{10}) a_{1}+(-2-\sqrt{10}) a_{2}+(2-2 \sqrt{2}-2 \sqrt{5}+\sqrt{10}) a_{3}\right), \\
& \frac{1}{2(2+\sqrt{10})}\left((-2+5 \sqrt{2}+2 \sqrt{5}-\sqrt{10}) a_{1}+(-10-2 \sqrt{10}) a_{2}+(8-5 \sqrt{2}-2 \sqrt{5}+\sqrt{10}) a_{3}\right), \\
& \left.\frac{1}{2+\sqrt{10}}\left((-2+2 \sqrt{2}+2 \sqrt{5}-\sqrt{10}) a_{1}+(-2-\sqrt{10}) a_{2}+(2-2 \sqrt{2}-2 \sqrt{5}+\sqrt{10}) a_{3}\right), a_{3}, a_{2}, a_{1}\right) .
\end{aligned}
$$

eigenvalue: -1

$M=2$

$$
\left(a_{0}, a_{1},\right) \propto\left((-\sqrt{2}+1) a_{1}, a_{1}\right)
$$

$M=6$

$$
\left(a_{0}, a_{1}, a_{2}, a_{3}, a_{4}, a_{5}\right) \propto\left(\frac{1}{2}\left(-\sqrt{6} a_{1}+(2-\sqrt{6}) a_{2}\right), a_{1}, a_{2}, \frac{1}{2}\left((2+\sqrt{6}) a_{1}-\sqrt{6} a_{2}\right), a_{2}, a_{1}\right)
$$


$M=10$

$$
\begin{aligned}
& \left(a_{0}, \ldots, a_{9}\right) \propto\left(\frac{1}{2}\left(-(1+\sqrt{2})(-1+\sqrt{5}) a_{1}-(-2+\sqrt{10}) a_{2}+(-1-\sqrt{2}+\sqrt{5}) a_{3}\right)\right. \\
& a_{1}, a_{2}, a_{3},(1+\sqrt{2}) a_{1}+a_{2}-(1+\sqrt{2}) a_{3}, \frac{1}{2}\left((1-\sqrt{5}) a_{1}-\sqrt{10} a_{2}+(1+\sqrt{5}+\sqrt{10}) a_{3}\right), \\
& \left.(1+\sqrt{2}) a_{1}+a_{2}-(1+\sqrt{2}) a_{3}, a_{3}, a_{2}, a_{1}\right)
\end{aligned}
$$

eigenvalue: $+i$

$M=2$ nothing,

$M=6$

$$
\left(a_{0}, a_{1}, a_{2}, a_{3}, a_{4}, a_{5}\right) \propto\left(0, a_{1},(-1+\sqrt{2}) a_{1}, 0,-(-1+\sqrt{2}) a_{1},-a_{1}\right),
$$

$M=10$

$$
\begin{aligned}
& \left(a_{0}, \ldots, a_{9}\right) \propto\left(0, a_{1}, a_{2},(-2+\sqrt{5+\sqrt{5}}) a_{1}+(-\sqrt{5}+\sqrt{5-\sqrt{5}}) a_{2},\right. \\
& \frac{1}{\sqrt{5-\sqrt{5}}}\left((-5+\sqrt{5}+\sqrt{25-5 \sqrt{5}}) a_{1}+2(-\sqrt{5}+\sqrt{5-\sqrt{5}}) a_{2}\right), 0, \\
& -\left(\frac{1}{\sqrt{5-\sqrt{5}}}\left((-5+\sqrt{5}+\sqrt{25-5 \sqrt{5}}) a_{1}+2(-\sqrt{5}+\sqrt{5-\sqrt{5}}) a_{2}\right)\right), \\
& \left.-\left((-2+\sqrt{5+\sqrt{5}}) a_{1}+(-\sqrt{5}+\sqrt{5-\sqrt{5}}) a_{2}\right),-a_{2},-a_{1}\right),
\end{aligned}
$$

eigenvalue: $-i$

$M=2$ nothing,

$M=6$

$$
\left(a_{0}, a_{1}, a_{2}, a_{3}, a_{4}, a_{5}\right) \propto\left(0, a_{1},(-1-\sqrt{2}) a_{1}, 0,-(-1-\sqrt{2}) a_{1},-a_{1}\right)
$$

$M=10$

$$
\begin{aligned}
& z\left(a_{0}, \ldots, a_{9}\right) \propto\left(0, a_{1}, a_{2},-(2+\sqrt{5+\sqrt{5}}) a_{1}-(\sqrt{5}+\sqrt{5-\sqrt{5}}) a_{2},\right. \\
& \frac{1}{\sqrt{5-\sqrt{5}}}\left((5-\sqrt{5}+\sqrt{25-5 \sqrt{5}}) a_{1}+2(\sqrt{5}+\sqrt{5-\sqrt{5}}) a_{2}\right), 0, \\
& -\left(\frac{1}{\sqrt{5-\sqrt{5}}}\left((5-\sqrt{5}+\sqrt{25-5 \sqrt{5}}) a_{1}+2(\sqrt{5}+\sqrt{5-\sqrt{5}}) a_{2}\right)\right), \\
& \left.-\left(-(2+\sqrt{5+\sqrt{5}}) a_{1}-(\sqrt{5}+\sqrt{5-\sqrt{5}}) a_{2}\right),-a_{2},-a_{1}\right),
\end{aligned}
$$

\section{2. $M=4 n$}

eigenvalue: +1

$M=4$

$$
\left(a_{0}, a_{1}, a_{2}, a_{3}\right) \propto\left(2 a_{1}+a_{2}, a_{1}, a_{2}, a_{1}\right),
$$


$M=8$

$$
\begin{aligned}
\left(a_{0}, \ldots, a_{7}\right) \propto & \left(\frac{1}{\sqrt{2}} a_{1}+(1+\sqrt{2}) a_{2}+\frac{1}{\sqrt{2}} a_{3}, a_{1}, a_{2}, a_{3},\right. \\
& \left.-\frac{1}{\sqrt{2}} a_{1}+(1+\sqrt{2}) a_{2}-\frac{1}{\sqrt{2}} a_{3}, a_{3}, a_{2}, a_{1}\right),
\end{aligned}
$$

$M=12$

$$
\begin{aligned}
& \left(a_{0}, \ldots, a_{11}\right) \propto\left(\frac{1}{2}\left((3+\sqrt{3}) a_{2}+2 \sqrt{3} a_{3}+(-1+\sqrt{3}) a_{4}\right),\right. \\
& a_{1}, a_{2}, a_{3}, a_{4},-a_{1}+\sqrt{3} a_{2}+2 a_{3}-\sqrt{3} a_{4}, \frac{1}{2}\left((-1+\sqrt{3}) a_{2}-2 \sqrt{3} a_{3}+(3+\sqrt{3}) a_{4}\right), \\
& \left.-a_{1}+\sqrt{3} a_{2}+2 a_{3}-\sqrt{3} a_{4}, a_{4}, a_{3}, a_{2}, a_{1}\right) .
\end{aligned}
$$

eigenvalue: -1

$M=4$

$$
\left(a_{0}, a_{1}, a_{2}, a_{3}\right) \propto\left(-a_{1}, a_{1}, a_{1}, a_{1}\right),
$$

$M=8$

$$
\begin{aligned}
& \left(a_{0}, \ldots, a_{7}\right) \propto\left(-\sqrt{2} a_{1}+(1-\sqrt{2}) a_{2}, a_{1}, a_{2}, a_{1},\right. \\
& \left.\sqrt{2} a_{1}+(1-\sqrt{2}) a_{2}, a_{1}, a_{2}, a_{1}\right)
\end{aligned}
$$

$M=12$

$$
\begin{aligned}
& \left(a_{0}, \ldots, a_{11}\right) \propto\left(\left(-1-\frac{1}{\sqrt{3}}\right) a_{1}+(1-\sqrt{3}) a_{2}+\left(1-\frac{2}{\sqrt{3}}\right) a_{3},\right. \\
& a_{1}, a_{2}, a_{3}, \frac{1}{3}\left(2 \sqrt{3} a_{1}+3 a_{2}-2 \sqrt{3} a_{3}\right), a_{1},(-1+\sqrt{3}) a_{1}+(1-\sqrt{3}) a_{2}+a_{3}, a_{1}, \\
& \left.\frac{1}{3}\left(2 \sqrt{3} a_{1}+3 a_{2}-2 \sqrt{3} a_{3}\right), a_{3}, a_{2}, a_{1}\right) .
\end{aligned}
$$

eigenvalue: $+i$

$M=4$

$$
\left(a_{0}, a_{1}, a_{2}, a_{3}\right) \propto\left(0, a_{1}, 0,-a_{1}\right),
$$

$M=8$

$$
\left(a_{0}, \ldots, a_{7}\right) \propto\left(0, a_{1}, a_{2}, a_{1}-\sqrt{2} a_{2}, 0,-\left(a_{1}-\sqrt{2} a_{2}\right),-a_{2},-a_{1}\right),
$$

$M=12$

$$
\begin{aligned}
& \left(a_{0}, \ldots, a_{11}\right) \propto\left(0, a_{1}, a_{2}, a_{3}, 2 a_{1}-a_{2}-(1+\sqrt{3}) a_{3},\right. \\
& \left.\quad-a_{1}-(-1-\sqrt{3}) a_{3}, 0, a_{1}+(-1-\sqrt{3}) a_{3},-2 a_{1}+a_{2}+(1+\sqrt{3}) a_{3},-a_{3},-a_{2},-a_{1}\right) .
\end{aligned}
$$


eigenvalue: $-i$

$M=4$ nothing,

$M=8$

$$
\left(a_{0}, \ldots, a_{7}\right) \propto\left(0, a_{1},-\sqrt{2} a_{1},-a_{1}, 0, a_{1}, \sqrt{2} a_{1},-a_{1}\right),
$$

$M=12$

$$
\begin{aligned}
& \left(a_{0}, \ldots, a_{11}\right) \propto\left(0, a_{1}, a_{2},-(1+\sqrt{3}) a_{1}-(1+\sqrt{3}) a_{2},\right. \\
& \left.a_{2}, a_{1}+2 a_{2}, 0,-a_{1}-2 a_{2},-a_{2},(1+\sqrt{3}) a_{1}+(1+\sqrt{3}) a_{2},-a_{2},-a_{1}\right) .
\end{aligned}
$$

\section{3. $M=4 n+3$}

eigenvalue: +1

$M=3$

$$
\left(a_{0}, a_{1}, a_{2}\right) \propto\left((\sqrt{3}+1) a_{1}, a_{1}, a_{1}\right),
$$

$M=7$

$$
\left(a_{0}, \ldots, a_{6}\right) \propto\left(L P_{0}\left(a_{1}, a_{2}\right), a_{1}, a_{2}, L P_{3}\left(a_{1}, a_{2}\right), L P_{3}\left(a_{1}, a_{2}\right), a_{2}, a_{1}\right),
$$

$M=11$

$$
\begin{aligned}
& \left(a_{0}, \ldots, a_{10}\right) \propto\left(L P_{0}\left(a_{1}, a_{2}, a_{3}\right), a_{1}, a_{2}, a_{3}, L P_{4}\left(a_{1}, a_{2}, a_{3}\right),\right. \\
& \left.L P_{5}\left(a_{1}, a_{2}, a_{3}\right), L P_{5}\left(a_{1}, a_{2}, a_{3}\right), L P_{4}\left(a_{1}, a_{2}, a_{3}\right), a_{3}, a_{2}, a_{1}\right) .
\end{aligned}
$$

eigenvalue: -1

$M=3$

$$
\left(a_{0}, a_{1}, a_{2}\right) \propto\left((-\sqrt{3}+1) a_{1}, a_{1}, a_{1}\right),
$$

$M=7$

$$
\left(a_{0}, \ldots, a_{6}\right) \propto\left(L P_{0}\left(a_{1}, a_{2}\right), a_{1}, a_{2}, L P_{3}\left(a_{1}, a_{2}\right), L P_{3}\left(a_{1}, a_{2}\right), a_{2}, a_{1}\right),
$$

$M=11$

$$
\begin{aligned}
& \left(a_{0}, \ldots, a_{10}\right) \propto\left(L P_{0}\left(a_{1}, a_{2}, a_{3}\right), a_{1}, a_{2}, a_{3}, L P_{4}\left(a_{1}, a_{2}, a_{3}\right),\right. \\
& \left.L P_{5}\left(a_{1}, a_{2}, a_{3}\right), L P_{5}\left(a_{1}, a_{2}, a_{3}\right), L P_{4}\left(a_{1}, a_{2}, a_{3}\right), a_{3}, a_{2}, a_{1}\right) .
\end{aligned}
$$

eigenvalue: $+i$

$M=3$

$$
\left(a_{0}, a_{1}, a_{2}\right) \propto\left(0, a_{1},-a_{1}\right)
$$

$M=7$

$$
\left(a_{0}, \ldots, a_{6}\right) \propto\left(0, a_{1}, a_{2}, L P_{3}\left(a_{1}, a_{2}\right),-L P_{3}\left(a_{1}, a_{2}\right),-a_{2},-a_{1}\right),
$$


$M=11$

$$
\begin{aligned}
& \left(a_{0}, \ldots, a_{10}\right) \propto\left(0, a_{1}, a_{2}, a_{3}, L P_{4}\left(a_{1}, a_{2}, a_{3}\right), L P_{5}\left(a_{1}, a_{2}, a_{3}\right),\right. \\
& \left.-L P_{5}\left(a_{1}, a_{2}, a_{3}\right),-L P_{4}\left(a_{1}, a_{2}, a_{3}\right),-a_{3},-a_{2},-a_{1}\right) .
\end{aligned}
$$

eigenvalue: $-i$

$M=3$ nothing,

$M=7$

$$
\left(a_{0}, \ldots, a_{6}\right) \propto\left(0, a_{1}, L P_{2}\left(a_{1}\right), L P_{3}\left(a_{1}\right),-L P_{3}\left(a_{1}\right),-L P_{2}\left(a_{1}\right),-a_{1}\right),
$$

$M=11$

$$
\begin{aligned}
& \left(a_{0}, \ldots, a_{10}\right) \propto\left(0, a_{1}, a_{2}, L P_{3}\left(a_{1}, a_{2}\right), L P_{4}\left(a_{1}, a_{2}\right), L P_{5}\left(a_{1}, a_{2}\right),\right. \\
& \left.-L P_{5}\left(a_{1}, a_{2}\right),-L P_{4}\left(a_{1}, a_{2}\right),-L P_{3}\left(a_{1}, a_{2}\right),-a_{2},-a_{1}\right),
\end{aligned}
$$

\section{4. $M=4 n+1$}

eigenvalue: +1

$M=5$

$\left(a_{0}, a_{1}, a_{2}, a_{3}, a_{4}\right) \propto\left(\frac{\sqrt{5}+1}{2}\left(a_{1}+a_{2}\right), a_{1}, a_{2}, a_{2}, a_{1}\right)$,

$M=9$

$$
\begin{aligned}
& \left(a_{0}, \ldots, a_{8}\right) \propto\left(L P_{0}\left(a_{1}, a_{2}, a_{3}\right), a_{1}, a_{2}, a_{3},\right. \\
& \left.L P_{4}\left(a_{1}, a_{2}\right), L P_{4}\left(a_{1}, a_{2}\right), a_{3}, a_{2}, a_{1}\right) .
\end{aligned}
$$

eigenvalue: -1

$M=5$

$$
\left(a_{0}, a_{1}, a_{2}, a_{3}, a_{4}\right) \propto\left((-\sqrt{5}+1) a_{1}, a_{1}, a_{1}, a_{1}, a_{1}\right),
$$

$M=9$

$\left(a_{0}, \ldots, a_{8}\right) \propto\left(L P_{0}\left(a_{1}, a_{2}\right), a_{1}, a_{2}, L P_{3}\left(a_{1}, a_{2}\right)\right.$,

$\left.L P_{4}\left(a_{1}, a_{2}\right), L P_{4}\left(a_{1}, a_{2}\right), a_{3}, a_{2}, a_{1}\right)$.

eigenvalue: $+i$

$M=5$

$\left(a_{0}, a_{1}, a_{2}, a_{3}, a_{4}\right) \propto\left(0, a_{1}, L P_{2}\left(a_{1}\right),-L P_{2}\left(a_{1}\right),-a_{1}\right)$, where

$$
L P_{2}\left(a_{1}\right)=-\frac{2}{1+\sqrt{5}-\sqrt{2(5+\sqrt{5})}} a_{1},
$$

$M=9$

$\left(a_{0}, \ldots, a_{8}\right) \propto\left(0, a_{1}, a_{2}, L P_{3}\left(a_{1}, a_{2}\right), L P_{4}\left(a_{1}, a_{2}\right)\right.$,

$\left.-L P_{4}\left(a_{1}, a_{2}\right),-L P_{3}\left(a_{1}, a_{2}\right),-a_{2},-a_{1}\right)$.

eigenvalue: $-i$

$M=5$

$\left(a_{0}, a_{1}, a_{2}, a_{3}, a_{4}\right) \propto\left(0, a_{1}, L P_{2}\left(a_{1}\right),-L P_{2}\left(a_{1}\right),-a_{1}\right)$,

where

$$
L P_{2}\left(a_{1}\right)=-\frac{2}{1+\sqrt{5}+\sqrt{2(5+\sqrt{5})}} a_{1},
$$

$M=9$

$\left(a_{0}, \ldots, a_{8}\right) \propto\left(0, a_{1}, a_{2}, L P_{3}\left(a_{1}, a_{2}\right), L P_{4}\left(a_{1}, a_{2}\right)\right.$,

$\left.-L P_{4}\left(a_{1}, a_{2}\right),-L P_{3}\left(a_{1}, a_{2}\right),-a_{2},-a_{1}\right)$. 
[1] C. Bachas, arXiv:hep-th/9503030.

[2] M. Berkooz, M. R. Douglas, and R. G. Leigh, Nucl. Phys. B480, 265 (1996).

[3] R. Blumenhagen, L. Goerlich, B. Kors, and D. Lust, J. High Energy Phys. 10 (2000) 006.

[4] C. Angelantonj, I. Antoniadis, E. Dudas, and A. Sagnotti, Phys. Lett. B 489, 223 (2000).

[5] D. Cremades, L. E. Ibanez, and F. Marchesano, J. High Energy Phys. 05 (2004) 079.

[6] H. Abe, K. S. Choi, T. Kobayashi, and H. Ohki, J. High Energy Phys. 06 (2009) 080.

[7] H. Abe, K. S. Choi, T. Kobayashi, and H. Ohki, Nucl. Phys. B820, 317 (2009).

[8] M. Berasaluce-Gonzalez, P. G. Camara, F. Marchesano, D. Regalado, and A. M. Uranga, J. High Energy Phys. 09 (2012) 059.

[9] Y. Hamada and T. Kobayashi, Prog. Theor. Phys. 128, 903 (2012).

[10] W. Buchmuller, M. Dierigl, E. Dudas, and J. Schweizer, J. High Energy Phys. 04 (2017) 052.

[11] D. M. Ghilencea and H. M. Lee, J. High Energy Phys. 06 (2017) 039.

[12] H. Abe, T. Kobayashi, H. Otsuka, and Y. Takano, J. High Energy Phys. 09 (2015) 056.

[13] H. Abe, T. Kobayashi, H. Otsuka, Y. Takano, and T. H. Tatsuishi, Prog. Theor. Exp. Phys. 2016, 053B01 (2016); Phys. Rev. D 94, 126020 (2016).

[14] H. Abe, T. Kobayashi, and H. Ohki, J. High Energy Phys. 09 (2008) 043.

[15] H. Abe, K. S. Choi, T. Kobayashi, and H. Ohki, Nucl. Phys. B814, 265 (2009).

[16] H. Abe, T. Kobayashi, H. Ohki, and K. Sumita, Nucl. Phys. B863, 1 (2012).

[17] H. Abe, T. Kobayashi, K. Sumita, and Y. Tatsuta, Phys. Rev. D 95, 015005 (2017).

[18] H. Abe, T. Kobayashi, and K. Sumita, Nucl. Phys. B911, 606 (2016).
[19] H. Abe, T. Kobayashi, K. Sumita, and S. Uemura, Phys. Rev. D 96, 026019 (2017).

[20] H. Abe, T. Kobayashi, H. Ohki, A. Oikawa, and K. Sumita, Nucl. Phys. B870, 30 (2013).

[21] H. Abe, T. Kobayashi, K. Sumita, and Y. Tatsuta, Phys. Rev. D 90, 105006 (2014).

[22] T. Kobayashi, Y. Tatsuta, and S. Uemura, Phys. Rev. D 93, 065029 (2016).

[23] T. Kobayashi, K. Nishiwaki, and Y. Tatsuta, J. High Energy Phys. 04 (2017) 080.

[24] W. Buchmuller, M. Dierigl, F. Ruehle, and J. Schweizer, Phys. Rev. D 92, 105031 (2015).

[25] W. Buchmuller, M. Dierigl, F. Ruehle, and J. Schweizer, Phys. Lett. B 750, 615 (2015).

[26] W. Buchmuller and J. Schweizer, Phys. Rev. D 95, 075024 (2017).

[27] M. Ishida, K. Nishiwaki, and Y. Tatsuta, Phys. Rev. D 95, 095036 (2017).

[28] T. H. Abe, Y. Fujimoto, T. Kobayashi, T. Miura, K. Nishiwaki, and M. Sakamoto, J. High Energy Phys. 01 (2014) 065.

[29] T.h. Abe, Y. Fujimoto, T. Kobayashi, T. Miura, K. Nishiwaki, and M. Sakamoto, Nucl. Phys. B890, 442 (2014).

[30] T. h. Abe, Y. Fujimoto, T. Kobayashi, T. Miura, K. Nishiwaki, M. Sakamoto, and Y. Tatsuta, Nucl. Phys. B894, 374 (2015).

[31] Y. Matsumoto and Y. Sakamura, Prog. Theor. Exp. Phys. 2016, 053B06 (2016).

[32] Y. Fujimoto, T. Kobayashi, K. Nishiwaki, M. Sakamoto, and Y. Tatsuta, Phys. Rev. D 94, 035031 (2016).

[33] T. Kobayashi and N. Ohtsubo, Phys. Lett. B 257, 56 (1991); Int. J. Mod. Phys. A 09, 87 (1994).

[34] T. Kobayashi, S. Nagamoto, and S. Uemura, Prog. Theor. Exp. Phys. 2017, 023B02 (2017).

[35] J. Lauer, J. Mas, and H. P. Nilles, Phys. Lett. B 226, 251 (1989); Nucl. Phys. B351, 353 (1991). 